\title{
Small-Scale Intelligent Vehicle Platform for Future Controls Course in the Application of Advanced Driving Assistance Systems
}

\section{Sarah De Rosier, California Polytechnic State University, San Luis Obispo}

Undergraduate mechanical engineering student interested in undergraduate research in the field of intelligent vehicles.

\section{Dominic Emilio Riccoboni, California Polytechnic State University, San Luis Obispo}

Dominic is a Mechanical Engineering senior interested in Mechatronics and Control Systems especially as the apply to Autonomous Vehicles, Space Technology, Robotics, and Biomedical Engineering.

\section{Mr. Paul Michael Rothhammer-Ruiz, California Polytechnic State University, San Luis Obispo}

Paul Rothhammer-Ruiz is a student at Cal Poly University in San Luis Obispo, CA. He is pursuing a Bachelor's degree in Mechanical Engineering with a concentration in Mechatronics. His interests include robotics, controls, and autonomous vehicles.

\section{Dr. Charles Birdsong, California Polytechnic State University, San Luis Obispo}

Charles Birdsong has expertise in automotive safety, vibrations, controls, signal processing, instrumentation, real-time control, active noise control, and dynamic system modeling. He received his B.S.M.E. at Cal Poly San Luis Obispo, and his M.S. and Ph.D. at Michigan State University, where he worked on active noise control applications for the automotive industry. He has worked in the vibration test and measurement industry helping to drive new technologies to market and working with industry to meet their emerging needs. He is currently a Professor at California Polytechnic State University at San Luis Obispo in the Department of Mechanical Engineering teaching dynamics, vibrations and controls. He is involved in several undergraduate and master's level multidisciplinary projects and interested in engineering education research. 
“Intelligent Vehicles:” Development of a new course for undergraduate engineering students 


\section{Introduction}

The field of intelligent vehicles, which includes Advanced Driving Assistance Systems (ADAS) and autonomous vehicle technologies, has grown rapidly in the last few years, creating a large demand for engineers with knowledge and experience in these technologies. Currently, there are 19 companies worldwide that are on track to have autonomous vehicle technology ready by 2020, including Toyota, Google, and Tesla [1]. The increased popularity in developing autonomous and intelligent vehicle technology has generated a need for thousands of jobs in the industry. However, the growing popularity of intelligent vehicles is outpacing the availability of engineers with enough experience to fill the positions. According to Sebastian Thrun, founder of Google’s self-driving car team, the global demand for engineers in the intelligent vehicle industry could be as high as 20,000 [2]. Because of this unmet need, intelligent vehicle powerhouses are paying big money for talent. In August of 2016, Uber purchased Otto, a selfdriving truck company with just 70 employees, for $\$ 680$ million. What Uber was willing to pay $\$ 680$ million for was not the technology, but the talent [3].

In response to this demand for talent, many universities are now offering courses in intelligent vehicles and intelligent vehicle control systems. One barrier to universities who wish to offer courses relating to intelligent vehicles, however, is the massive expense of developing and testing full-scale intelligent vehicles. University of Michigan, for example, spent \$10 million developing an entire 32-acre mock city, Mcity, in order to serve as a proving ground for their intelligent vehicles [4]. University of Michigan went to the expense of constructing this facility in order to provide a safe, controlled environment for the testing of their intelligent vehicles. Unfortunately, many universities across the country lack the funds and space for such an endeavor. One solution to this problem would be to develop a small-scale vehicle that could be used as a testing platform for intelligent vehicle technologies, without incurring the expense and safety hazards of a full-scale vehicle. This paper presents a design for such a small-scale intelligent vehicle (SSIV) to be used as a learning platform for a course at California Polytechnic State University of San Luis Obispo (Cal Poly). The platform is shown below in Figure 1. The course and platform are being developed by students at Cal Poly, in collaboration with a professor who has a vision for the course. What follows is a description of how the course will prepare students for careers in industry; a look at similar courses at other universities; an overview of the course; a summary of the SSIV development; and a plan for evaluating the course. 


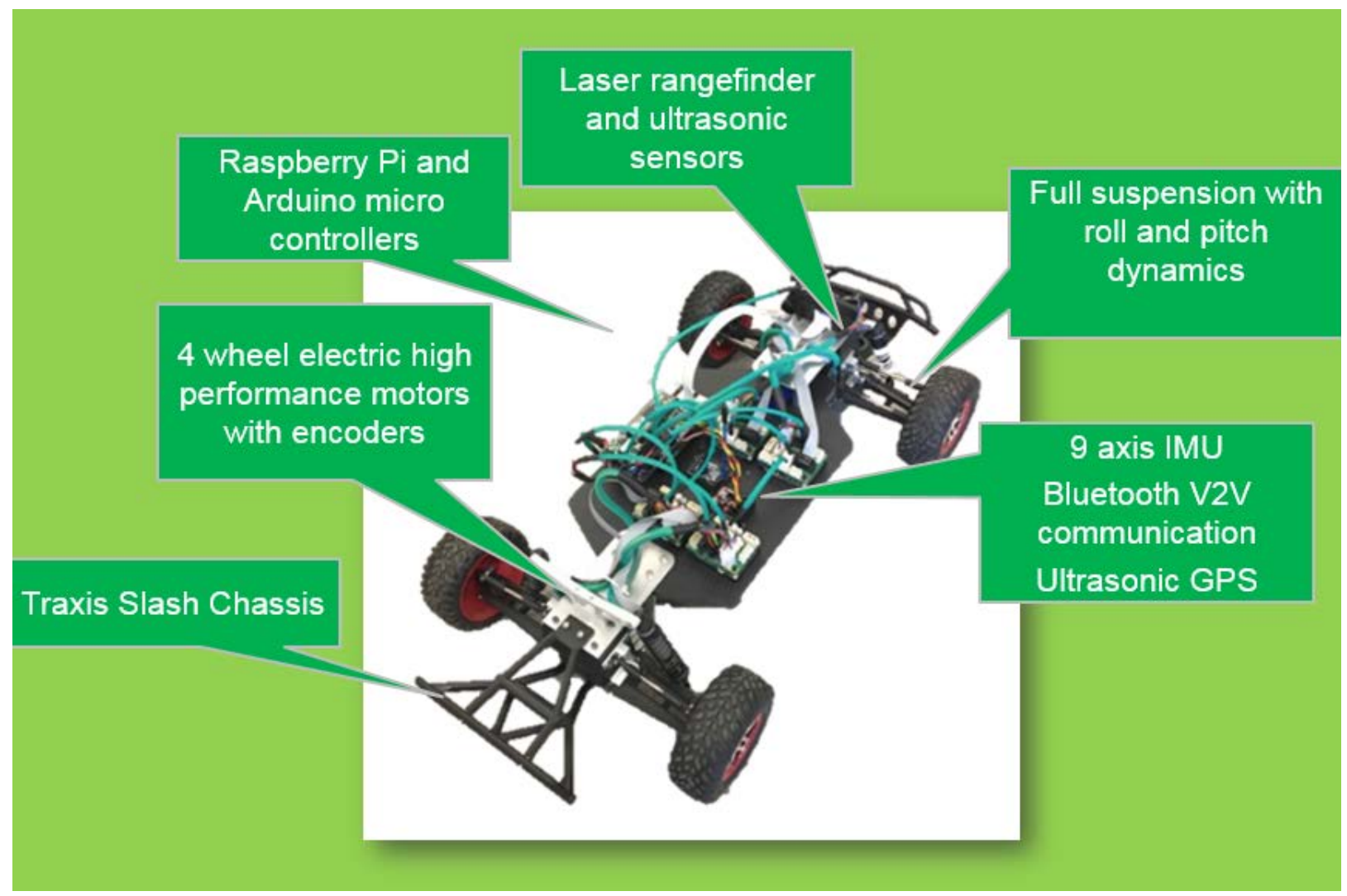

Figure 1. The Small Scale Intelligent Vehicle (SSIV) in its current state of development.

Tailoring the Course to Meet Industry Needs

At Cal Poly, we wanted to make sure that our course in intelligent vehicles would effectively prepare students for a career in the industry. To better understand what areas of expertise industry professionals were looking for, we asked engineers working with ADAS features at Daimler Trucks North America what subjects they would like incoming entry level engineers at their company to be familiar with. Vehicle dynamics, linear controls, Controller Area Network (CAN) protocol, computer vision, autonomous navigation (including object tracking, lane recognition, path planning, and sign recognition), neural networks, and machine learning were the subjects mentioned most frequently. They also suggested a need for familiarity with radar, digital cameras, ultrasonic sensors, GPS, accelerometers, gyroscopes, LiDAR, and encoders, as well as sensor fusion and Kalman filters [5,6].

\section{Approaches to Teaching Intelligent Vehicles at Other Universities}

At Cal Poly, we want to introduce a course that will cover topics such as the ones listed above applied to the field of intelligent vehicles. The course will use the SSIV as a platform for the lab portion of the course, so students can apply what they learn to a physical system. There are a couple of universities that are currently implementing a setup such as this one. MIT has developed a small-scale vehicle that they call RACECAR (Rapid Autonomous ComplexEnvironment Competing Ackermann-steering Robots) [7]. This vehicle is controlled by an NVIDIA GPU and can run on-board machine learning algorithms. While MIT is also offering courses on intelligent vehicles and control, the RACECAR is not used as a lab platform for any 
of those courses. University of California, Berkeley (UC Berkeley) has also designed the Berkeley Autonomous Race Car (BARC), which can perform lane keeping, obstacle avoidance, and autonomous drifting [8]. They are currently developing a course focused on introducing vehicle dynamics and implementing controls onto the small-scale vehicle.

\section{The Course}

We are proposing a course that covers vehicle dynamics, computer vision, sensor fusion, filtering, signal processing, autonomous navigation, and controls. Our course will be accessible to students of all engineering backgrounds_-including electrical engineers, computer engineers, and mechanical engineers. In order to be as inclusive as possible to these students from different backgrounds, all programming will be done in Mathworks Simulink, a graphical programming environment that doesn't require familiarity with languages such as C++ and Python. Simulink is also used in controls applications throughout the automotive industry by companies such as General Motors, Volkswagen, Daimler, Nissan, and Toyota, so experience with the program will help prepare students for work in the field of intelligent vehicles [9].

The course will be offered as a weekly three-hour lecture and a three-hour lab. The lecture and lab topics are shown in Table 1 below. In the lab, students will have an opportunity to apply topics introduced in lecture.

Table 1. Lecture and Lab Topics Per Week

\begin{tabular}{|l|l|l|}
\hline Week & Lecture & Lab \\
\hline 1 & $\begin{array}{l}\text { Overview of state-of-the-art } \\
\text { intelligent vehicles }\end{array}$ & $\begin{array}{l}\text { Introduction to the small scale intelligent vehicle; } \\
\text { simple steering and speed command programming; } \\
\text { implement and monitor lane change maneuver }\end{array}$ \\
\hline 2 & Vehicle dynamic modeling & $\begin{array}{l}\text { Monitor and record IMU and encoder data; digital } \\
\text { filtering of IMU data }\end{array}$ \\
\hline 3 & Sensors and Image Processing & $\begin{array}{l}\text { Lane detection from digital camera data, and lane- } \\
\text { keeping algorithm }\end{array}$ \\
\hline 4 & $\begin{array}{l}\text { Longitudinal acceleration } \\
\text { control }\end{array}$ & Adaptive cruise control algorithm \\
\hline 5 & Lateral acceleration control & Stability control algorithm \\
\hline 6 & Stability control & $\begin{array}{l}\text { Term project - each team of 2-4 students will } \\
\text { propose a project that can be analyzed, modeled, } \\
\text { programmed and tested in four weeks. They will } \\
\text { write a final technical report and give a presentation } \\
\text { illustrating the idea, method and results. }\end{array}$ \\
\hline 7 & $\begin{array}{l}\text { Signal Processing-digital } \\
\text { filtering }\end{array}$ & \\
\hline 8 & Sensor fusion & Autonomous vehicles \\
\hline 10 & Project presentations & \\
\hline
\end{tabular}


For each lab, students will be asked to design a controller that will allow the SSIV to perform a specific behavior such as lane keeping, adaptive cruise control, acceleration control, or stability control. The students will be provided with an incomplete Simulink model of the system and asked to fill in the missing components to create a working controller before designing control parameters for the model. To prove that their design works, students will run tests on the SSIV and demonstrate through sensor data that the expected response was achieved. They will also analyze their results to determine the effects of external disturbance and noise on the system. In the second half of the course, students will develop a term project in which they extend the abilities of the SSIV. Students could also choose from a variety of additional sensor technologies to integrate onto the vehicle. The instructor will guide students to keep the term project scope within reason for the time limits of the course. Students will then be asked to demonstrate the results of their efforts, provide testing data, and present on their design process.

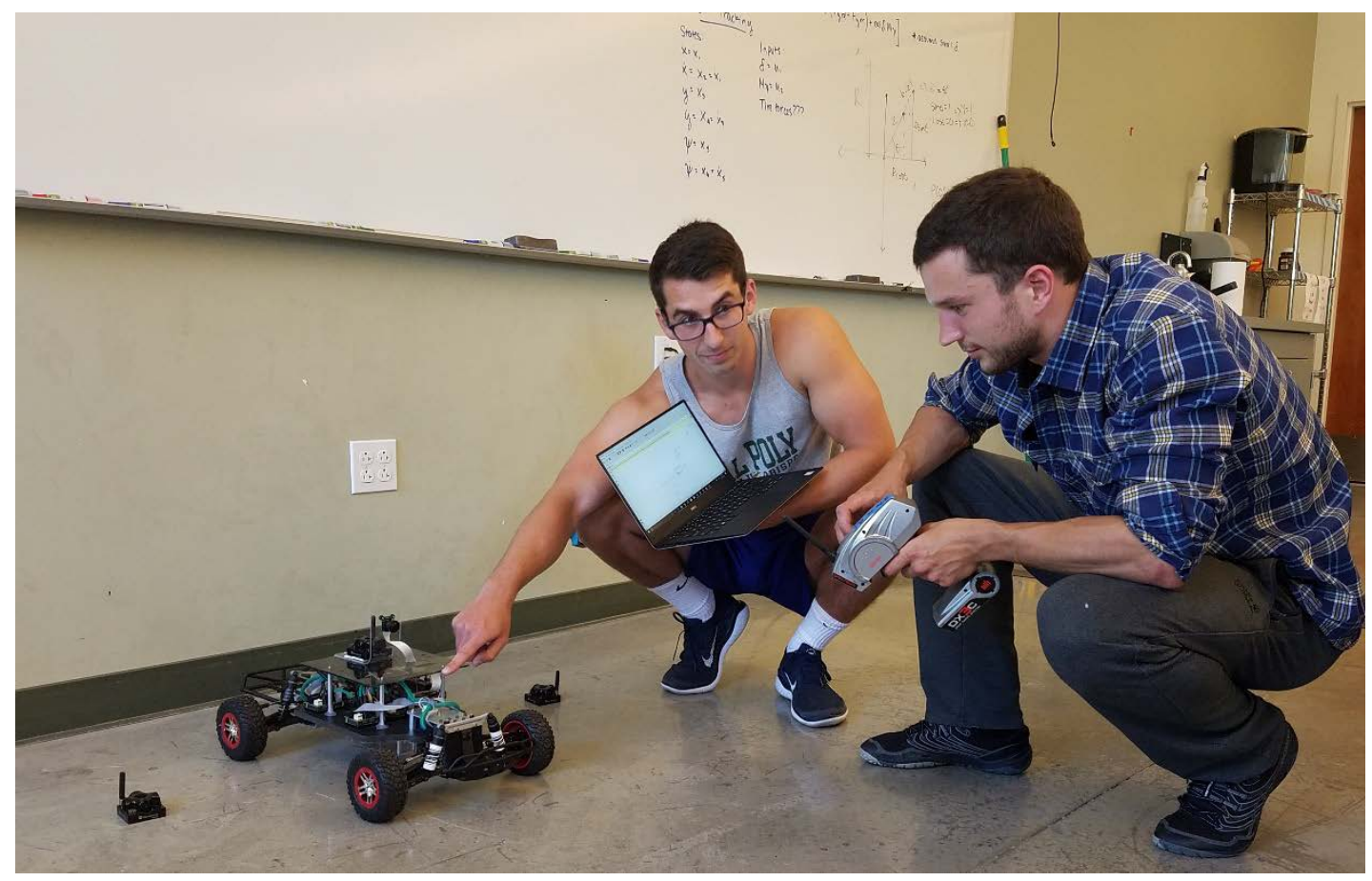

Figure 2. Students performing an experiment on the SSIV in a lab.

In addition to understanding the control algorithm implementation, students will gain many of the skills currently in demand in industry, including vehicle dynamics, computer vision, sensor fusion, filtering, and autonomous navigation. Students will also get to interface with sensors currently used in industry, including radar, digital cameras, ultrasonic sensors, GPS, accelerometers, gyroscopes, LiDAR, and encoders. The learning outcomes of the course are listed below.

- Understand the state-of-the-art of intelligent vehicles and the benefits and challenges of this technology. 
- Derive, implement and use a numerical model of a vehicle with inputs of motor torque, steering and braking and outputs of longitudinal, lateral and yaw velocities. The model will include mass properties, tire forces, slip angles and dimensions.

- Operate a small scale intelligent vehicle by programming in the Matlab/Simulink environment. This will include implementing command signals, measuring sensor signals and applying closed-loop feedback techniques.

- Select appropriate sensors for different applications and understand the benefits and limitations in their use.

- Apply closed-loop feedback control techniques for advanced driver assistance systems in simulation, including speed control, distance control, lane keeping, stability control, and collision avoidance.

- Implement signal processing algorithms to filter sensor signals and integrate multiple sensors for situational awareness.

- Apply modern control theory to the field of intelligent vehicles, including state-space modeling, state feedback, observability, controllability, and observer-controller.

The purpose of this course is to familiarize students with these various topics and how they are applied to intelligent vehicles. The SSIV platform will serve as a hands-on tool that trains students to appropriately model the dynamic system of a car, prototype and test controllers for ADAS features, utilize various sensors, and filter and process sensor data.

\section{The Design of the SSIV}

The platform was specifically developed with the purpose of modeling the dynamics of a fullscale car. We decided to start with a $1 / 10^{\text {th }}$-scale RC car so we would not need to design and manufacture mechanical components such as bumpers, A-arms, u-joints, tie rods, and drive shaft. However, one issue with using an RC car to run control algorithms is that most RC cars only have one motor per car, and therefore do not have the ability to run control algorithms such as stability control and torque vectoring. We decided to design a vehicle chassis with space for four motors that utilized existing RC car components. Figure 1 below shows the SSIV in its current state.

Because the SSIV must be capable of implementing control algorithms designed in Simulink, this imposes a number of constraints on the computational hardware that can be used. To achieve this capability, we are using a Raspberry Pi single-board computer with the MathWorks Raspberry Pi support package. Using this hardware-software combination, controllers designed in Simulink can be deployed onto the vehicle with little effort by the students. To produce the signals necessary to control the vehicle, the Raspberry-Pi/Simulink control loop sends actuations and receives sensor data via digital communication protocol. Sensors providing controller feedback are an integral part of the design. For example, the rangefinder and ultrasonic sensors will measure the distance to objects in front of the car for the adaptive cruise control lab. The Inertial Measurement Unit (IMU) measures the orientation and acceleration, which will be used primarily in the stability control and lane keeping labs. Four brushless DC (BLDC) motors, one at each wheel, propel the vehicle, and can be configured to emulate different drive trains seen on 
cars. The shafts of these motors are fitted with rotary encoders to provide the feedback necessary for control. Four independent motor controllers will drive the motors to meet torque setpoints. The sensors and motor drivers that will be integrated on the car will provide adequate feedback once proper filtering has been introduced. An example Simulink control algorithm implementation is shown below in Figure 3.

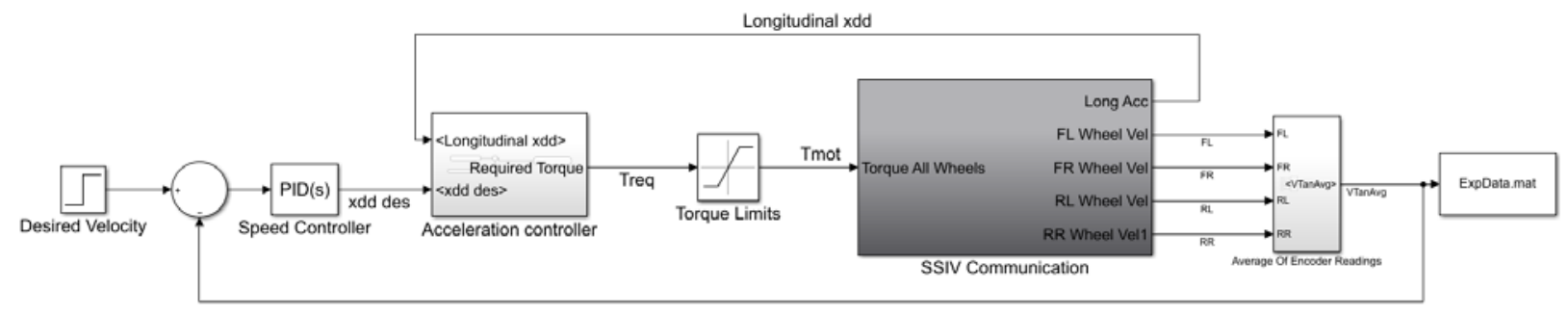

Figure 3. A simple cruise control algorithm designed in Simulink to run on the platform.

\section{The Vision}

We hope that this SSIV will also be used by student researchers and graduate students interested in testing new technologies or expanding its capabilities. In addition, we hope to share this platform with other institutions and universities to encourage more universities to offer hands-on undergraduate courses in intelligent vehicles. The parts and CAD files necessary to build the vehicle, along with assembly instructions, will be posted online. Because we plan to share this design with other universities, it is important that the platform is produced at the lowest cost possible. Currently, we have designed the platform to use many purchased parts, and to take advantage of low-cost manufacturing processes such as 3D printing. The current platform uses 4 independent compact high torque motors and 4 high performance motor controllers. We are working on reducing the cost of the system by selecting alternative motors and designing a stripped down motor controller board customized for this application. The target price for one vehicle is $\$ 1,000$ which should allow a course to procure a set of several vehicles to be used in the lab course.

The effectiveness of this course will be evaluated twofold: the students will be evaluated on how their conceptual understanding of the topics presented in the course improved, and the students will be given an opportunity to provide feedback regarding the structure and content of the course. In order to evaluate their conceptual understanding, students will be given the same survey at the beginning and end of the course. The survey will look very similar to that presented below.

1. List three benefits of intelligent vehicles

2. Describe the slip angle and how it relates to vehicle dynamics.

3. What are the typical state variables in a vehicle dynamic model?

4. What sensor would you use to measure:

a. Tire position

b. Distance between leading and following car 
c. Yaw rate

5. What signal processing technique can be used to combine a GPS sensor and a radar sensor to estimate a vehicle's position?

6. When is an observer needed in a control system?

7. What are the limitations of the linear tire model?

8. What are some of the limitations of using a single camera and hand-crafted image processing techniques for lane detection?

9. Why do we have to run an open loop velocity response for the Adaptive Cruise Controller lab?

10. What are the two components of a typical vehicle stability controller?

How students improve in their responses to this survey will be one measure by which the course is evaluated. Students will be able to provide feedback where they evaluate the course based upon criteria such as their interest in the subject matter, whether they liked how the subject matter was presented, and how they feel their understanding of the topics improved throughout the course. The feedback survey, given at the end of the course, will look very similar to that presented below.

1. How was the pace of the course? Circle one:

Too fast Just right $\quad$ Too slow

1. How have your views on intelligent vehicles changed throughout this course?

2. Are you interested in pursuing a career in intelligent vehicles?

3. What was an aspect of intelligent vehicles that was not covered in the course that you think should be included?

4. Were there any topics related to intelligent vehicles that you felt did not belong in the course?

5. Do you feel that you came into this course with adequate prerequisite knowledge?

6. Did the structure of the lab and lecture make sense? Do you feel that the lab aided your understanding of concepts presented in lecture, and vice versa?

7. Do you have any recommendations regarding the order in which topics are presented?

It is our hope that students will come out of this course with hands-on experience working with intelligent vehicle technologies that will prepare them for a job in the industry. Over the next five months, we will continue to develop the capabilities of the vehicle platform to meet the technical needs of the course. We will also develop the courseware in collaboration with the professor of the course. The course is scheduled to be offered in the 2018-2019 academic year. 
[1] Muoio, Danielle. "These 19 Companies Are Racing to Put Driverless Cars on the Road by 2020.” Business Insider, Business Insider, 15 July 2016, www.businessinsider.com/googleapple-tesla-race-to-develop-driverless-cars-by-2020-2016-7.

[2] “What Does the Future of Self-Driving Cars Hold for Tech Talent?” Paysa Blog, Paysa, 28 Aug. 2017, www.paysa.com/blog/2017/01/09/what-does-the-future-of-self-driving-cars-hold-fortech-talent/.

[3] Woodall, Bernie. “Uber Buys Self-Driving Truck Startup Otto; Teams with Volvo.” Reuters, Thomson Reuters, 18 Aug. 2016, www.reuters.com/article/us-uber-tech-volvo-otto/uber-buysself-driving-truck-startup-otto-teams-with-volvo-idUSKCN10T1TR.

[4] “Mcity Test Facility.” Mcity, University of Michigan, mcity.umich.edu/our-work/mcity-testfacility/.

[5] Noxon, Nikola. Personal interview. 18 December 2017.

[6] Trabant, Carl. Personal interview. 21 December 2017.

[7] "Mit-Racecar." GitHub, MIT, github.com/mit-racecar/.

[8] "Berkeley Autonomous Race Car.” BARC, University of California Berkeley, www.barcproject.com/.

[9] The MathWorks in the Automotive Industry. The MathWorks in the Automotive Industry, The MathWorks, 2008. 